\title{
Transferring face-to-face sessions to virtual sessions in surgical education: a survey-based assessment of a single academic general surgery program
}

\author{
Mauricio Gonzalez-Urquijo (D) · David E. Gonzalez-Hinojosa · Javier Rojas-Mendez · Mario Rodarte-Shade
}

Received: 27 December 2020 / Accepted: 15 January 2021 / Published online: 9 February 2021

(c) Springer-Verlag GmbH, AT part of Springer Nature 2021

\begin{abstract}
Summary
Background The purpose of this survey-based study was to evaluate the implementation of virtual learning in a single academic general surgery program, compared with the well-established face-to-face academic curriculum used before.

Methods From April 2020 to the present, virtual sessions were created via Zoom Videos Communications, Inc. (San Jose, CA, USA). A survey composed of 15 questions about the perceived quality and utility of the virtual sessions was developed. The survey was sent out to all general surgery residents of a general surgery program in November of 2020.

Results All residents enrolled in the program answered the survey, i.e., $22(73.3 \%)$ men and 8 (26.7\%) women with $6(20.2 \%)$ residents per year (PGY 1-PGY 5). Over half of residents $(n=17,56.7 \%)$ felt similar academic performance during online sessions when compared to the older model. Perceptions of the level of organization of academic sessions increased during the online model ( $n=20,66.7 \%)$. Twenty $(66.7 \%)$ residents agreed it was easier to attend sessions during the online model. Fourteen (46.7\%) residents reported their attendance to the sessions increased, and 14 (46.7\%)
\end{abstract}

\footnotetext{
M. Gonzalez-Urquijo $(\bowtie) \cdot$ D. E. Gonzalez-Hinojosa • J. Rojas-Mendez · M. Rodarte-Shade Tecnologico de Monterrey, School of Medicine and Health Sciences, Dr. Ignacio Morones Prieto O 3000, 64710 Monterrey, Nuevo León, Mexico mauriciogzzu@gmail.com
}

D. E. Gonzalez-Hinojosa d.hinojosa94@gmail.com

J. Rojas-Mendez

Jrojas_mendez@hotmail.com

M. Rodarte-Shade

marioshade@hotmail.com residents would prefer this modality after the pandemic is over, with $8(26.7 \%)$ being neutral about it. Conclusion This study, to our knowledge, is the first to survey general surgery residents about the transition from a face-to-face curriculum to an e-learning curriculum. The demonstrated effectiveness of the transition from face-to-face academic activities to virtual activities makes it a feasible tool for graduate medical education programs to adjust to a virtual model.

Keywords e-Learning $\cdot$ Teaching $\cdot$ Academic performance $\cdot$ Postgraduate education $\cdot$ Medical education

\section{Main novel aspects}

This study, to our knowledge, is the first to survey general surgery residents about the transition from a faceto-face curriculum to an e-learning curriculum, and the first to compared objectively academic grade scores between both models.

In our study, we found no differences when comparing mean scores between virtual and face-to-face courses.

Our experience of transitioning to online learning demonstrated that virtual activities are a feasible tool for graduate medical education programs.

\section{Introduction}

The outbreak of the current coronavirus disease 2019 (COVID-19) pandemic generated profound effects on surgical education and training. One of these effects was the incorporation of virtual learning to maintain academic curriculums while avoiding large gatherings. Most medical schools have withdrawn their students from in-hospital clerkships; however, this did not happen in postmedical graduates or residents, 
since they form part of the task-force in the fight against COVID-19 [1]. COVID-19 has highlighted the significance of telemedicine. From online didactics to virtual rounds to teleclinic, bringing telemedicine into a new light will help significantly in the postCOVID era [2].

It is well known that the greatest burden for resident training is the decreased time spent in the operating room, refining, and practicing surgical techniques [2, 3]. Nevertheless, this paper focuses on the resident's perception about clinical academic sessions transferred into a virtual method, and not on how it affected their day-to-day clinical surgical practice.

To our knowledge, there are no studies evaluating the emotional, intellectual, and academic changes in surgical residents since implementation of the virtual sessions, and most of the studies are either editorials on the virtual new models or are focused only on medical students, or in the change faced by surgical residencies in the decreased exposure to surgical procedures [4].

The COVID-19 pandemic has increased the utilization of virtual learning at our institution; however, to our knowledge, the efficacy of its use and satisfaction of the alumni has not been studied. The purpose of the current study was to evaluate resident perspectives on the use of virtual learning.

In light of the above, the purpose of this surveybased study was to evaluate the implementation of virtual learning in a single academic general surgery program during the COVID-19 pandemic, compared with the well-established face-to-face academic curriculum used before the pandemic.

\section{Materials and methods}

The study was performed with prior consideration from Internal Review Board and in accordance to institutional, regional, national and international laws regarding clinical research, including norms established by the Helsinki Conference in 1964 and its revision in 2012 as it does local national regulations, including adherence to Article 17 of the Mexican General Health Law (Ley General de Salud en Materia de Investigación para la Salud).

From April 2020 to the present, virtual sessions were created via Zoom Videos Communications, Inc. (San Jose, CA, USA). In our program, we have a structured educational curriculum, which includes daily one hour of morning teaching ward rounds, and one hour a week of the following activities: academic class per residency year, journal club, tumor board, morbidity and mortality session, clinical case discussion, anatomy seminar, and clinical lecture performed by an expert in a specific field, among other sessions with different disciplines such as internal medicine, pathology, and radiology. Sessions totaled a maximum of $15 \mathrm{~h}$ per week, and a minimum of $10 \mathrm{~h}$ per week of virtual sessions.
A survey composed of 15 questions was developed by the program director, and the chief of residents from the general surgery program of Tecnologico de Monterrey, School of Medicine and Health Sciences. After a literature review and remote discussions, the most suitable questions were selected to evaluate the use of virtual learning in our program before and during the pandemic. Four surgical professors evaluated the final version of the survey and agreed that the final version of the questionnaire was clear and unambiguous. The survey was conducted exclusively in the English language. Supporting data and answers are available upon request from the corresponding author.

The survey was sent to all general surgery residents of a single academic general surgery program on 1 November 2020 via e-mail with a cover letter and link to access the survey on Google Forms (Google LLC, Mountain View, CA, USA). Completed questionnaires were received between 2 and 10 November 2020. Responders could answer the survey only once. Participation in the survey was voluntary and anonymous but demographic data including gender, age, and year of residency were gathered. Questions were about the perceived quality and utility of the virtual sessions; answers were stratified into negative (disagree), neutral, and positive (agree).

Data were analyzed in SPSS v25 (IBM, Armonk, NY, USA). Kolmogorov-Smirnov tests were used to assess normality of distribution of variables. Parametric continuous variables are expressed as means and standard deviations and were analyzed through t-student and analysis of variance (ANOVA) where applicable, using Tukey's post hoc testing. Nonparametric continuous variables were tested using Mann-Whitney $U$-test or Kruskall Wallis and expressed as medians and ranges. Frequencies are reported as absolute counts and percentages and were tested using either Chi-square or Fisher's exact. Academic grading in our institution is based on a scale of $0-100$, and it is evaluated per semester (September-February, and March-August), where a final exam of residents by residency year (PGY) is individually taken (grade A), and a mean score of all clinical sessions given by each resident (grade B), having a total of two different final grades. Grades from prepandemic performance (September-February 2019) were compared with pandemic academic performance (March-August 2020) through paired t-student testing.

A $P$ value $<0.05$ was considered statistically significant.

\section{Results}

Overall, 30 residents consented to participation, representing $100 \%$ of residents enrolled in the program. These were made up of $22(73.3 \%)$ men and 8 (26.7\%) women. There were $6(20.2 \%)$ residents per year (PGY $1-P G Y 5)$. Mean resident age was $28.43 \pm 2.12$. 


\section{Perception of virtual sessions}

Overall, $23(73.3 \%)$ residents agreed skills are needed for effective online presentations being different from face-to-face presentations and residents were mostly neutral about online presentations being more valuable academically than traditional presentations $(n=16,53.3 \%)$.

Over half of residents $(n=17,56.7 \%)$ felt similar academic performance during online sessions when compared to the older model. Eight $(26.7 \%)$ residents believed that online presentations were of more value than face-to-face presentations. Perceptions of the level of organization of academic sessions increased during the online model ( $n=20,66.7 \%)$; furthermore, $76.7 \%(n=23)$ of residents agreed that it was easier to understand the academic sessions during e-learning, compared with the conventional model.

Twenty $(66.7 \%)$ residents agreed it was easier to attend sessions during the online model. Fourteen $(46.7 \%)$ residents reported their attendance to the sessions increased, and $14(46.7 \%)$ residents would prefer this modality after the pandemic is over, with 8 $(26.7 \%)$ being neutral about it. Half $(n=15,50 \%)$ of the residents believe their participation during sessions increased and $14(46.7 \%)$ residents believed the academic level of these presentations is of a higher academic level than before. Most residents $(n=18$, $60.0 \%$ ) agreed interpretation of imaging studies was easier during online presentations. Residents' perception of ease of participation was split, as only 11 (36.7\%) felt it was easier to participate, and 9 (30\%) remained neutral about it.

Fourteen $(46.7 \%)$ residents felt equal fatigue compared to the prior presentation model, while residents were pretty split about their perceived burnout during the pandemic, with 10 (33.3\%) feeling less fatigued, 8 (26.7\%) feeling equally and $12(40.0 \%)$ feeling more fatigued. Overall findings are displayed in Table 1.

\section{Academic performance}

Virtual model mean scores of grade A was of $84.37 \pm 6.76$ and grade $B$ of $90.00 \pm 7.55$, compared with the conventional model mean scores of grade A of $88.16 \pm 8.47$ and grade $B$ of $92.45 \pm 7.95$, which were not statically different ( $P=0.76$ and $P=0.212$, respectively), thus, suggesting the academic model has been effective at blunting educational impacts that the pandemic might have on resident learning.

\section{Discussion}

This cross-sectional online survey about virtual academic activities showed that residents are adapting positively to the new method of learning since there were more positive responses than negative ones. During the pandemic, there was a switch to distance learning, with Zoom being our preferred platform for tele-education. It should be noted that our program managed to maintain the teaching hours for theoretical training at a satisfactory level because the surgical training was dramatically decreased during the pandemic due to the suspension of elective surgeries, as in most countries. Before the pandemic, due to diverse clinical activities (e.g., local and international rotations), the attendance of residents to most sessions was low.

Higher-learning institutions have implemented virtual learning to their curriculum since it is cost-effective, accessible, and flexible in terms of time and location. The main interest in virtual education is the interactive communication it permits. This method enables instructors to receive immediate feedback from participants, allowing them to adjust their presentations accordingly [5]. Two basic modules of virtual learning exist: synchronous, which requires all participants to be available at the same time, enabling communication and interaction between the educator and trainees, and asynchronous, where participants

Table 1 Survey of academic perception of virtual academic sessions from general surgery residents

\begin{tabular}{|c|c|c|c|}
\hline Question & $\begin{array}{l}\text { Disagree } \\
N(\%)\end{array}$ & $\begin{array}{l}\text { Neutral } \\
N(\%)\end{array}$ & $\begin{array}{l}\text { Agreed } \\
N(\%)\end{array}$ \\
\hline Presentation skills are different for online presentations & $3(10.0)$ & $5(16.7)$ & $22(73.3)$ \\
\hline Virtual sessions have been more useful for my training as a resident, than before & $11(36.7)$ & $16(53.3)$ & $3(10.0)$ \\
\hline My academic performance has increased & $6(20.0)$ & $17(56.7)$ & $7(23.3)$ \\
\hline Online presentations help me better understand the content of the session & $10(33.3)$ & $12(40.0)$ & $8(26.7)$ \\
\hline Academic organization is better compared with before & $4(13.3)$ & $6(20.0)$ & $20(66.7)$ \\
\hline I find it easier to attend online sessions & $5(16.7)$ & $2(6.7)$ & $23(76.7)$ \\
\hline My attendance has increased & $5(16.7)$ & $11(36.7)$ & $14(46.7)$ \\
\hline Online sessions should continue even after the pandemic is over & $8(26.7)$ & $8(26.7)$ & $14(46.7)$ \\
\hline My participation in the sessions has increased & $5(16.7)$ & $10(33.3)$ & $15(50.0)$ \\
\hline The academic level of the sessions is higher & $8(26.7)$ & $8(26.7)$ & $14(46.7)$ \\
\hline I find it easier to see and evaluate image studies & $6(20)$ & $6(20)$ & $14(46.7)$ \\
\hline It's easier to participate during online sessions & $10(33)$ & $9(30)$ & $11(36.7)$ \\
\hline I've felt more tired during online sessions & $4(13.3)$ & $14(46.7)$ & $12(40)$ \\
\hline I've felt more tired during the pandemic & $12(40)$ & $8(26.7)$ & $10(33.3)$ \\
\hline
\end{tabular}


can access educational content at any time, attending a web-based training course [6].

Some studies indicate that residents and medical students who use e-learning programs are more satisfied with their learning experience, compared with those who use traditional teaching methods [7]. One of the main reasons is that virtual learning platforms allowed the learner to go through the content once seen in a live session, to be accessed at any time, at any speed, backward and forward, with increasing flexibility and personalized lessons [8]. Furthermore, virtual learning has been shown to have lower costs due to reduced travel, reduced institutional infrastructure, and it gives the opportunity to cross borders by inviting renowned experts in the field [9]. On the other hand, an important limitation of virtual learning is the technical problems faced during online activities, such as requiring more data transmission than the regular amount, with loss of connection or delay and decrease of fluency reported. Another minor challenge is the necessity to obtain a suitable atmosphere in the resident's home, avoiding the many interruptions that are usually not present in face-to-face presentations [7].

Essilfie et al. [10] who surveyed 168 orthopedic surgical residents found a higher gratification with e-learning compared with in-person learning and found the former to be more beneficial to their daily travel time. However, they felt they were more likely to pay attention to in-person learning, as distractions might be more readily accessible. Likewise, Machado et al. [11] reported the adversities of maintaining focus and concentration while sitting in front of a screen. In our study $53.3 \%$ of residents were more positive about online presentations, reporting that they are of more academic value than traditional ones. However, contrary to the aforementioned studies, $76.7 \%$ of our residents reported not having concentration issues and being able to better understand the academic sessions by virtual learning.

Another benefit of virtual learning is that these sessions have allowed residents to engage with surgeons from other counties, with larger pools of experienced tutors and geographic flexibility, as geographic influence has been shown to affect how surgeons approach common problems [10, 12]. For example, in Mexico there is no formal acute care and trauma surgery program/curriculum. Thus, for a whole week, we had online sessions, courses, and journal clubs, with an acute care and trauma surgeon from San Francisco, California. Technology has allowed us to bridge gaps in knowledge, ultimately benefiting patients.

It is important to note that virtual learning does not replace instructor-led teaching entirely. In surgery, direct supervision at the place of work by a professor is still required [9]. There are dynamic aspects that take place during face-to-face interactions that cannot be replicated virtually. Some of these aspects include learning to publicly speak, maintaining eye contact with the public, picking up nonverbal signs, and measuring audience interest and engagement. Moreover, the ability to network and the development of significant relations are more likely to occur during face-toface sessions [10, 13].

A brief report published by Lara et al. [14] documented the novel use of a teleconference objective structured clinical examination (teleOSCE) during the current pandemic. The results of this study indicated that for the 49 medical students who participated, there was no difference in mean score (mean difference $-1.1 ; 95 \%$ confidence interval $[\mathrm{CI}]-2.8$ to 0.7 ; $p=0.2$ ) or failure (rate difference $2 \%$; $95 \% \mathrm{CI}-0.7$ to $10.7 \% ; p=0.06$ ) between face-to-face and teleOSCEs. They suggested that this form of assessment to be an effective and reliable method of testing, which could be explored further in the future. Likewise, in our study when comparing mean scores between virtual and face-to-face courses, we found no difference between them. We also think that e-learning has arrived abruptly and has arrived to stay.

We believe our experience of transitioning to online learning adds to the current evidence and may prove useful to academic programs in similar situations. In addition, advantages identified during the pandemic are a worthwhile carryover to traditional education once the pandemic is resolved.

There are several limitations to this survey study. The number of respondents is relatively low and may not be representative of the entire country. Nonetheless, academic activities between Mexican general surgery programs vary, so making a multicenter-program survey would be biased.

In conclusion, this study, to our knowledge, is the first to survey general surgery residents about the transition from a face-to-face curriculum to an e-learning curriculum, and the first to compared objectively academic grade scores between both models. The demonstrated effectiveness of the transition from face-to-face academic activities to virtual activities makes it a feasible tool for graduate medical education programs to adjust to a virtual model. Institutions should actively seek to adapt to situations, like the one we are living in, to accelerate student learning. Future study endeavors could assess attendings'/professors' perceptions of e-learning and compared them with the ones of the trainees.

Author Contribution Mauricio Gonzalez-Urquijo-conception of the work, drafting of manuscript, final approval and agreement for the accountability of work. David E. Gonzalez-Hinojosa-data interpretation, revising the manuscript, final approval and agreement for the accountability of work. Javier Rojas-Mendez-data interpretation, revising the manuscript, final approval and agreement for the accountability of work. Mario Rodarte-Shade-data interpretation, revising the manuscript, final approval and agreement for the accountability of work. 


\section{Compliance with ethical guidelines}

Conflict of interest M. Gonzalez-Urquijo, D. E. GonzalezHinojosa, J. Rojas-Mendez and M. Rodarte-Shade declare that they have no competing interests.

Ethical standards All procedures performed in studies involving human participants were in accordance with the ethical standards of Tecnologico de Monterrey ethics committee and have therefore been performed in accordance with the ethical standards laid down in the 1964 Declaration of Helsinki and its later amendments. Informed consent was obtained from all individual participants included in the study. A copy of the written consent is available for review by the Editor-inChief of this journal on request.

\section{References}

1. Ehrlich H, McKenney M, Elkbuli A. We asked the experts: virtual learning in surgical education during the COVID-19 pandemic - shaping the future of surgical education and training. World J Surg. 2020;44(7):2053-5. https://doi.org/ 10.1007/s00268-020-05574-3.

2. Aziz H, James T, Remulla D, et al. Effect of COVID-19 on surgical training across the United States: a national survey of general surgery residents. J Surg Educ. 2020; https:// doi. org/10.1016/j.jsurg.2020.07.037.

3. Ellison EC, Spanknebel K, Stain SC, Shabahang MM, Matthews JB, Debas HT, et al. Impact of the COVID-19 Pandemic on Surgical Training and Learner Well-Being: Report of a Survey of General Surgery and Other Surgical Specialty Educators. J Am Coll Surg [Internet]. 2020;231(6):613-26. https://doi.org/10.1016/j.jamcollsurg.2020.08.766.

4. Wilcha R-J. Effectiveness of virtual medical teaching during the COVID-19 crisis: systematic review. JMIR Med Educ. 2020;6:1-16. https://doi.org/10.2196/20963.

5. FairbanksJ. What' shappeningdistance educationfornurse practitioners: a partial solution. J Am Acad Nurse Pract. 1995;7(10):499-503.
6. Curran VR. Education and practice. J Telemed Telecare. 2006;12:57-63. https://doi.org/10.1016/S01406736(49)91151-4.

7. Figueroa F, Figueroa D, Calvo-Mena R, et al. Orthopedic surgery residents' perception of online education in their programs during the COVID-19 pandemic: should it be maintained after the crisis? acta Orthop. 2020; https://doi. org/10.1080/17453674.2020.1776461.

8. Tarpada SP, Morris MT, Burton DA. E-learning in orthopedic surgery training: a systematic review. J Orthop. 2016;13(4):425-30. https://doi.org/10.1016/j.jor.2016.09. 004.

9. Aryal KR, Pereira J. E learning in surgery. Indian J Surg. 2014;76(6):487-93. https://doi.org/10.1007/s12262-0141092-8.

10. Essilfie AA, Hurley ET, Strauss EJ, et al. Resident, fellow, and attending perception of E-learning during the COVID19 pandemic and implications on future orthopaedic education. J Am Acad Orthop Surg. 2020;29:e860-e4. https:// doi.org/10.5435/JAAOS-D-20-00579.

11. Machado RA, Bonan PRF, da Perez DEC, et al. I am having trouble keeping up with virtual teaching activities: reflections in the COVID-19 era. Clinics. 2020;75:e1945. https://doi.org/10.6061/clinics/2020/e1945.

12. Hau H-M, Weitz J, Bork U. Impact of the COVID-19 pandemic on student and resident teaching and training in surgical oncology. JClin Med. 2020;9(11):3431. https://doi. org/10.3390/jcm9113431.

13. Atreya A, Acharya J. Distant virtual medical education during COVID-19: half a loaf of bread. Clin Teach. 2020;17(4):418-9. https:// doi.org/10.1111/tct.13185.

14. Lara S, Foster CW, Hawks M, et al. Remote assessment of clinical skills during COVID-19: a virtual, high-stakes, summative pediatric objective structured clinical examination. Acad Pediatr. 2020;20(6):760-1. https://doi.org/10.1016/j. acap.2020.05.029.

Publisher's Note Springer Nature remains neutral with regard to jurisdictional claims in published maps and institutional affiliations. 